\title{
Intelligent Agents as Cells of Immunological Memory
}

\author{
Krzysztof Cetnarowicz ${ }^{1}$, Gabriel Rojek ${ }^{2}$, and Rafał Pokrywka ${ }^{3}$ \\ 1 Institute of Computer Science \\ AGH University of Science and Technology \\ al. Mickiewicza 30, 30-059 Kraków, Poland \\ cetnar@agh.edu.pl \\ 2 Department of Computer Science in Industry \\ AGH University of Science and Technology \\ al. Mickiewicza 30, 30-059 Kraków, Poland \\ rojek@agh.edu.pl \\ 3 IBM SWG Laboratory ${ }^{\star}$ \\ ul. Armii Krajowej 18, 30-150 Kraków, Poland \\ rpokrywka@gmail.com
}

\begin{abstract}
Application of mechanisms of immune memory in the computer security domain allows to increase performance of certain class of security systems that are based on detection of attacks without a priori knowledge of attack's technique. Immune memory should enable the system to memorise once encountered attacks and prevent it together with its consequences in the future. The use of agent technologies gives new possibilities in the management of stored attack's patterns - patterns of obsolete attacks should be deleted but those of new and frequent should be maintained and generalised. In this paper ideas from agent technology and immune memory domain are introduced into computer security, tested and discussed.
\end{abstract}

\section{Introduction}

Work presented here is a part of research on Host Intrusion Detection System (HIDS) which is based on anomaly detection. This kind of security systems are usually placed in key nodes of network infrastructure. The system detects anomalies by comparing normal and current behaviour of monitored processes. This approach allows to detect an attack without a priori knowledge of attack's technique. To model the behaviour of a process the Markov model with variable order is used.

Here we would like to focus on mechanisms which will enable the system to record attacks and to use this recorded information to prevent the same or similar attacks in the future. In addition detection ought to be faster and the level of false alarms should decrease. All those merits should be achieved by the

\footnotetext{
* This paper is related to my knowledge and activities before I joined IBM.
} 
realisation of mechanism similar to the natural immune memory. Agent technologies should allow to maintain stable in number population of recognisable patterns. The population should also dynamically adapt to all current threats by removal of agents responsible for detection of obsolete attacks and maintaining those responsible for detection of new and frequent ones. Agents use simple neural network to make decisions about particular threat. This should also enable them to generalise and detect similar attacks to those recorded. Similar attacks are those with only slightly changed technique.

\section{Overview of the Natural Immune Memory}

The human immune system is designed to protect an organism against pathogens. The most important elements of the system are lymphocytes which in great number circulate through the body and detect foreign cells. Basically there are two types of lymphocytes: $\mathrm{T}$ and $\mathrm{B}$. They both cooperate in detection and elimination of pathogens. They also cooperate in creation of immune memory which we would like to model.

Immune memory is created during the primary immune response. This response is usually slow and has low intensity. The secondary immune response is initiated by memory cells and is very fast, intensive and often runs without any clinical symptoms of infection. Additionally the fact that it is triggered by infection with new, but similar to previously seen, pathogens means that immune memory is associative.

The primary response consists of several steps. First the lymphocyte of type $B$ detects newly encountered pathogen. Then it receives confirmation of positive detection from lymphocyte T. This confirmation is called co-stimulation. Next it is activated and starts to mutate with high rate until it becomes specific to this one particular kind of pathogens. After the completion of this step the specific Bcell proliferate and differentiate into plasma cells and memory cells. The former secrete antibodies which participate in elimination of the pathogen, the later memorise the pathogen. Memory cells do not need any co-stimulation to become activated and to mount the secondary response, during which they rapidly proliferate and differentiate into plasma cells. This results in a fast increase of the number of antibodies and a very fast elimination of the threat. A detailed description of the entire immune system and can be found in [1], [4] and [5].

\section{Mechanisms of Anomaly Detection}

In this section a basic information about the HIDS used in our researches into immune memory is presented. The system is based on anomaly detection in behaviour of processes working on a given host. Behaviour is represented by sequences of system calls. Grounds for this choice can be found in [2] and [3].

Analysis of conditional entropy of the sequences suggests that it is reasonable to choose one of the statistical model which incorporates conditional probability to build a profile of these sequences. Here we have chosen the Markov chain with 
variable order. This model provides a probability of the next system call having seen previous $n$. In this case $n$ is a length of history and depends on the actual context - hence variable order. The profile is created during the training phase from the sequences of system calls representing normal behaviour according to the algorithm presented in [7]. An overview of Markov models can be found in [6].

Executing the next system call is a single step associated with the probability provided by the Markov model. During the monitoring phase this probability is mapped to some amount of penalty points. It is done through a penalty function $\xi:[0,1] \rightarrow \mathbb{R}$. To be useful this function should give a large amount of penalty points for probability equal to 0 and small amount if the probability is close to 1. In our research the following function was used:

$$
\xi(x)=\frac{a}{x+b}+c .
$$

The $a$ parameter controls how convex the function is, $b$ and $c$ parameters modifies the amount of points the function returns for a given probability. All three parameters must be carefully chosen. In each step the computed penalty points are added to a penalty account $\Xi$. To limit aggregation of history the penalty account is also multiplied by dumping factor $\zeta<1(3)$.

$$
\begin{aligned}
& \Xi_{0}=0 \\
& \Xi_{i+1}=\zeta\left(\Xi_{i}+\xi_{i, i+1}\right)
\end{aligned}
$$

where $\xi_{i, i+1}$ is the amount of the penalty points computed when going from $i$-th step to $(i+1)$-th step. If the penalty account exceeds a certain threshold $\tau$ an alarm is fired. Figure 1 presents example plot of the penalty account and the threshold. From observations it can be concluded that each process has its own characteristic plot of behaviour what is a very important feature.

Subsequent values of the penalty account form a signal which is unique for particular process and is also unique for an anomaly. It is reasonable though to remember and analyse this signal and through this recognise intrusions. Anomaly detector described in this section is a first-line analyser. It fires an alarm whenever signal exceeds the threshold.

The main disadvantage of all anomaly detection methods is the possibility of false alarms. In addition detection is often too slow and attack or exploit could have been already executed. In this case any alarm has only informative value. Immune memory can speed up the moment of detection and thus possibly prevent the consequences of an attack. It can also lower the level of false alarms with time.

\section{Intelligent Immunological Memory}

The main idea of presented research is to realise memory cells as intelligent agents which are able to remember part of the process' signal (subsequent values of the penalty account). The memory agent is born in the moment of raising an 


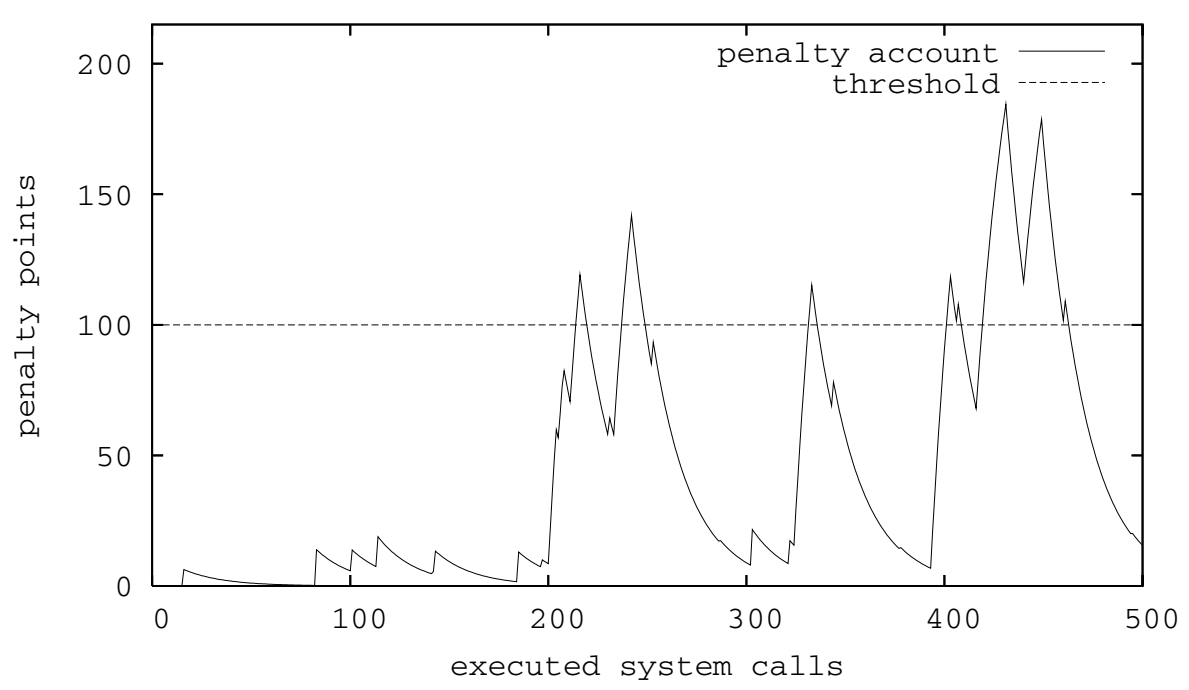

Fig. 1. Example plot of the penalty account and the threshold

alarm by the anomaly detector. Because of the possibility of false alarms this newly created agent needs a second signal to become fully functional. This signal comes from an administrator who must confirm that detected anomaly is really a threat. It is a kind of co-stimulation like in the natural immune system. This is the only place where human intervention is required. After the administrator's decision the agent is turned into one of the two types:

- anomaly specific — in case there was an anomaly (co-stimulation). The goal of this agent is to remember particular pattern of an attack and detect its future appearances.

- anergic - in case it was an false alarm (no co-stimulation). This agent's aim is to remember false alarm pattern and prevent future appearances of this false alarm.

Agents of both types use neural network to recognise patterns in the process' signal. Neural network also enables the agent to recognise similar patterns to that remembered. The anomaly specific agent has simply its neural network trained to remember a particular attack's pattern. Anomaly specific agents are able to initiate adequate response such as killing the process or tracking all execve calls. Anergic agents are specific to patterns of false alarms and if they manage to detect something no response should be initiated (there is an analogical mechanism in the natural immune system). Anergic agents are able to lower the level of false alarms with time. For IDS systems it is crucial to keep false alarm rate as low as possible. This is because usually the number of normal events in a system is significantly larger than the number of abnormal events. Normal events are therefore analysed more often and even if false alarm rate is low most raised 
alarms turn out to be false. This problem is known as base rate fallacy and is directly implicated by Bayes' theorem. More details can be found in [8].

\subsection{Life Energy}

To maintain a stable in number population of agents and also to build a population which can dynamically adapt to current threats an idea of life energy is introduced. At the beginning an agent receives some fixed amount of life energy $E_{p}$. In each step the energy is decreased by 1 . There is also determined a certain probability of death of the agent, which depends on the actual level of the agent's life energy. The probability is given by the following function:

$$
p(x)= \begin{cases}-\frac{1}{E_{s}}+1 & \text { for } x<E_{s} \\ 0 & \text { for } x \geq E_{s}\end{cases}
$$

where $E_{s}$ is an arbitrarily chosen value. When the agent happens to detect something its life energy increases also by arbitrarily chosen value $E_{a}$. This mechanism makes it possible to delete agents which detect obsolete patterns. It also keeps alive those agents responsible for detection of frequently occurring patterns.

\subsection{Neural Network}

The ADALINE (ADAptive LINear Element) neuron is capable of recognising the most similar patterns to the remembered one what is a key feature for our purposes. Since the agent is (in this version) responsible only for detection of one particular pattern its neural network consists of one ADALINE neuron. The pattern is represented by a vector of $m$ last values of the process signal. The neuron also has $m$ inputs and $m$ input weights. For now it is assumed that the length of the pattern is constant.

The activation of the neuron is given by the following formula:

$$
y=\sum_{i=1}^{m} w_{i} x i
$$

where $w_{i}$ is the $\mathrm{i}$-th weight and $x_{i}$ is the $\mathrm{i}$-th input value. Inputs are often written simply as vector $X$ and weights as vector $W$. From properties of ADALINE the more input vector $X$ is similar to the weight vector $W$ the greater the activation [9].

The neuron is learned through delta-rule which is an iterative process of adjusting the vector of weights according to the formula below:

$$
\begin{aligned}
W^{\prime} & =W+\eta \delta X \\
\delta & =t-y
\end{aligned}
$$

where $\eta$ is the learning ratio, $t$ is the target value that the neuron should give for this particular input vector $X, y$ is the actual neuron's output for the input 
vector $X$. The agent's decision is positive when the current output is close enough to the target value, for example when it is $s=99 \%$ of the $t$. On the choice of $s$ depends agent's capabilities of generalisation. The $s$ parameter defines also patterns similarity. In other words the agent groups similar patterns of similar anomalies.

\section{$5 \quad$ Experimental Results}

Datasets for tests were obtained from publicly available repository prepared by researchers from Computer Science Department, University of New Mexico [10].

The datasets consists of normal and anomalous traces of privileged processes. A trace is a sequence of system calls only.

To measure how fast an anomaly is detected a MTFA (Mean Time to First Alarm) metric is used. The MTFA is defined as:

$$
\frac{\sum_{\rho \in S_{\mathrm{an}}} \operatorname{alarm}_{1}(\rho)}{\left|S_{\mathrm{an}}\right|}
$$

where $S_{\text {an }}$ is a suite of anomalous traces, $\left|S_{\text {an }}\right|$ is the size of the suite and $\operatorname{alarm}_{1}(\rho)$ is a number of system call when the trace $\rho$ was first identified as anomalous. The rate of false alarms is measured simply as the ratio between the number of times normal trace is identified as anomalous and the number of times a trace was classified correctly.

In performed tests traces of sendmail were used. The normal database of this program consists of 346 traces and 1799764 system calls. Anomalous database consists of 25 traces 6755 system calls. There are traces of decode and sunsendmailcp exploits available and also an error condition - forwarding loop. There was 518229 normal system calls used in the training phase and 1281535 and all anomalous calls in the monitoring phase.

The testing phase begins when there is trained anomaly detector and empty agents population. The parameters for the anomaly detector were as follows: $\zeta=0.95, \tau=40$. For the penalty function it was assumed that in each step the maximal amount of penalty points was 15 and for probability 1 the function returned 0 . Therefore the $b$ and $c$ parameters could be computed. The $a$ parameter was set to 20. This parameter combination gave the best results and was chosen empirically. The population of anergic agents is created during runs through normal database and theirs $E_{p}$ was chosen adequately to the size of the normal traces. Anomaly specific agents are created in the first and next runs through anomalous database. The $E_{s}$ parameter was set to $\frac{E_{p}}{3}$ and $E_{a}=E_{p}$. Those parameters were also chosen empirically so that to achieve a stable agents population. The size of pattern was $m=30$.

Tests have shown that specific memory agents detects anomalous traces faster than anomaly detector alone. It can be seen in a decrease in MTFA (Fig. 2). After the first run through normal database anergic agents have lowered falsealarm rate down to 0.0 (Fig. 3). Figure 5 presents a growth of the population of anergic agents with time. The number of agents doesn't fall down since all 


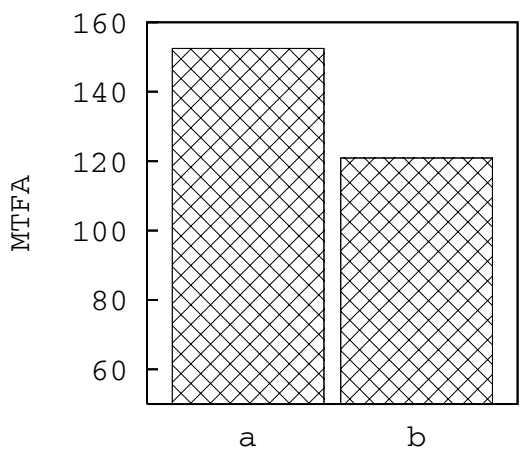

Fig. 2. Decrease in MTFA after the use of specific agents. The 'a' column refers to the result when no specific agents were used, 'b' when agents were used.

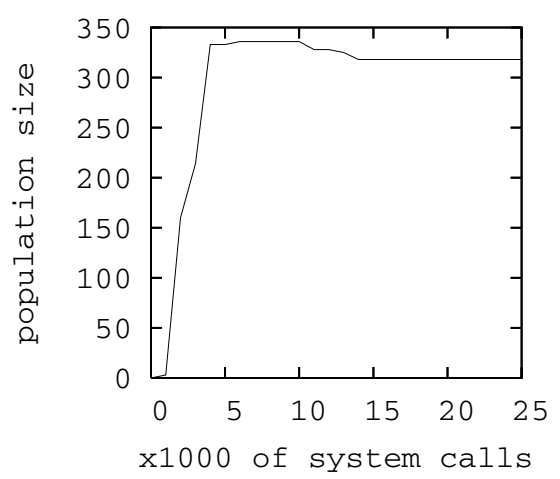

Fig. 4. The growth of population of specific agents

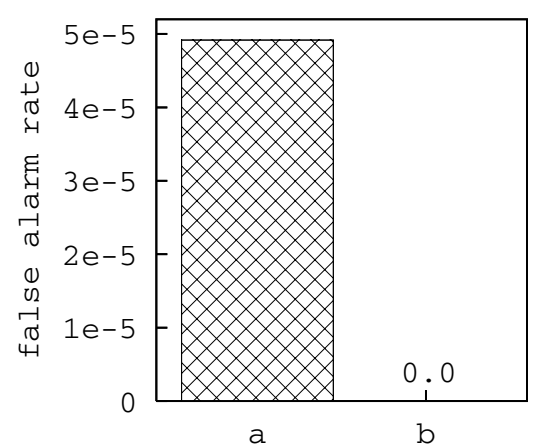

Fig. 3. Decrease in the level of false alarms when anergic agents are used. The 'a' column refers to the result without and 'b' with anergic agents.

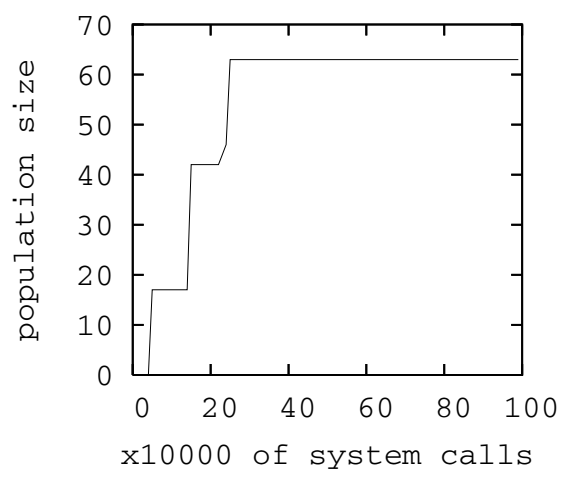

Fig. 5. The growth of population of anergic agents

patterns responsible for false alarms are present in the test suite. The growth of population of specific agents is presented on Fig. 4. At first all anomalous traces were included in the test suite what is reflected in the rapid growth and stabilisation of the population. Next, after two runs through the anomalous database, the traces of the decode exploit were removed. It can be seen that the population has shrunk adequately to the new situation.

\section{Conclusions and Further Work}

In performed tests it has been shown that memory agents are useful as an improvement of intrusion detection systems based on anomaly detection. They may help to prevent intrusions when a particular patch has yet to be developed. 
Memory agents speed up the detection and lower false-alarm rate. Anergic agents are also useful when there is some change in program behaviour caused by, for example, library update. Other advantages include the reduction in amount of administrator's work and the adaptation of the agents population to the external conditions through mechanism of life-energy. However the disadvantage is that in real implementation the computer resources consumption is very high.

Tests presented here provide only a proof of concept and further work is needed. There are a few directions in which further research can go. First another potential benefits from using agent technologies must be mentioned. For example an ability to immunise other hosts in a LAN/WAN area. In this case a property of mobility must first be given to an agent. Additional benefits like better generalisation and prediction may be achieved by using specialized neural networks used in pattern recognition in financial analysis. This may result in further improve of MTFA. More investigation into identification by behaviour is also needed.

\section{References}

1. Hofmeyr, S.A.: An Interpretative Introduction to the Immune System. To Appear in the Design Principles for the Immune System and other Distributed Autonomous Systems, Oxford University Press (2000)

2. Forrest, S., Hofmeyr, S.A., Somayaji, A., Longstaff, T.: A Sense of Self for Unix Processes. In IEEE Symposium on Security and Privacy (1996) pages 120-128

3. Hofmeyr, S.A., Forrest, S., Somayaji, A.: Intrusion Detection Using Sequences of System Calls. Journal of Computer Security Vol. 6 (1998) pages 151-180

4. Wierzchoń, S.T.: Sztuczne systemy immunologiczne. Teoria i zastosowania. (in polish), EXIT, Warszawa (2001)

5. Kim, J. W.: Integrating Artificial Immune Algorithms for Intrusion Detection. PhD Thesis, Department of Computer Science, University College London (2002)

6. Bengio, Y.: Markovian Models for Sequential Data. Neural Computing Surveys 2 (1999) pages 129-162

7. Ron, D., Singer, Y., Tishby, N.: The Power of Amnesia: Learning Probabilistic Automata with Variable Memory Length. Machine Learning, 25(2-3) (1996) pages $117-149$

8. Axelsson, S.: The Base-Rate Fallacy and the Difficulty of Intrusion Detection. ACM Transactions on Information and System Security (TISSEC) 3 (2000) pages 186-205

9. Tadeusiewicz, R.: Sieci Neuronowe. (in polish), Wyd. 2, Akademicka Oficyna Wydawnicza RM, Warszawa (1993)

10. Data sets available on-line at http://www.cs.unm.edu/ immsec/systemcalls.htm. 2006 